\title{
Translational medicine: path to personalized and
}

\section{public health}

“Community integration establishes a basis for bidirectionality in translational research, in which neighborhood representatives shape the research agenda and inform the prioritization of questions, individualizing medical research that is aligned with specific community needs."

\section{KEYWORDS: individualized medicine " personalized medicine " team science - translational medicine}

Individualized medicine aims to provide personalized solutions based on patient-specific molecular mechanisms of disease by transforming traditional healthcare practices that are applied to the population. Indeed, the individual variability in disease manifestation and treatment outcome, exemplars of challenges in today's medicine, impede optimal patient management and compromise population health. This variability reflects current limitations in the knowledge base of disease processes and in the inadequate delivery of most promising healthcare solutions. Molecular sciences, including the evolving science of biomarkers, have catalyzed the development of diagnostic and therapeutic platforms tailored to the individual patient profile, paving the way for the deployment of individualized medicine approaches that address human variation and individualized medical need [1]. This next generation of medicine is largely enabled by technological breakthroughs that have led to advanced resolutions of disease states, guided development of prognostic discriminators of disease variability and selection of treatment response predictors [2]. Patient-centric algorithms, grounded in the individual's genetic makeup, are emerging as the flagship of contemporary medicine [3]. The implementation of personalized practices, offering unprecedented opportunities for earlier diagnosis, effective prevention and disease cures, present a new era in medicine, and has the opportunity to transform the standard of care for individual patients and populations.

\section{Translational algorithm}

Healthcare 2020 is conceptualized on the integration of discovery, development, application and dissemination sciences [4]. Discovery has been advanced by the innovation fueled by the 'omics', informational and systems biology revolution, enabling the development of personalized diagnostics and therapeutics, molecular markers of disease prognosis, and clinical predictors of therapeutic responses, guiding management algorithms optimized for patients and communities $[5,6]$. In fact, deconvolution of corrupted biological pathways in disease and translation of patient-specific molecular mechanisms into tailored management have begun to extend the reach of individualized medicine from principles to practice [7]. To this end, the science of molecular biomarkers represents a critical cornerstone in the transformation of current models of patient care towards increasingly preemptive, prognostic and predictive medical paradigms. Spanning across medical and surgical specialties, translational medicine is thereby poised to identify the most effective science-based healthcare decision systems that align clinical care with individual patient and community needs. The emergence of translational science, as the key paradigm to optimize medical as well as surgical products and services emanating from the intersection of discovery science and healthcare delivery, highlights the unifying framework that bridges the continuum of knowledge creation and deployment, converting fundamental discoveries to human application, advancing the information into clinical practice, disseminating best clinical practices into communities and, ultimately, modifying the behavior of populations to improve global health [8].

\section{Translational prototype}

A prototype of individualized medicine, regenerative medicine, has begun to define a new perspective of future clinical practice [9,10]. The

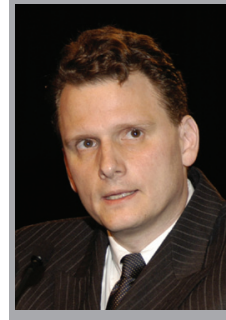

Andre Terzic

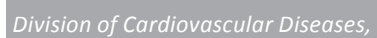
Departments of Medicine, Molecular Pharmacology \& Experimental Therapeutics \& Medical Genetics, Mayo Clinic, 200 First Street SW, Rochester, MN 55905, USA

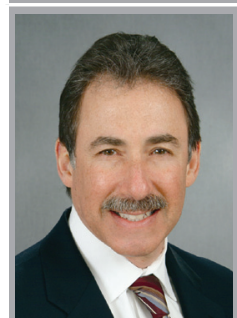

Scott A Waldman

Author for correspondence: Department of Pharmacology \& Experimental Therapeutics, Division of Clinical Pharmacology, Department of Medicine, Thomas Jefferson University, 132 South, 10th Street, 1170 Main, PA 19107, USA scott.waldman@jefferson.edu 
US Department of Health and Human Services report '2020: A New Vision - A Future for Regenerative Medicine' highlights that regenerative medicine is the vanguard of 21st century healthcare [101]. Patients and society increasingly expect that regenerative medicine will lead, ultimately, to the repair of diseased organs, injured tissues and congenital anomalies. From pioneering success with bone marrow transplants for select hematological disorders, which are now the standard of care, to the most recent advances in bioengineered stem cell platforms that provide unlimited sources of autologous pluripotent progenitors, and broaden the scope of individualized diagnosis and therapy [11,12], the NIH [102] and National Academies [103] recognize regenerative medicine as a most promising core component of modern medical practice. Without the contribution of personalized products and services that are emerging from regenerative medicine technology, experts caution that the healthcare sector will face an escalation in inefficient treatments and a rising global cost [13]. Aimed at functional restoration of damaged tissues, not a mere abatement or moderation of symptoms, regenerative medicine offers a 'disruptive innovation' strategy, uniquely poised to add value and transform healthcare by providing tailored, curative solutions for the unmet needs of patients [14]. Indeed, tissue repair might provide a sustained therapeutic advantage in a spectrum of conditions, ranging from congenital diseases to acquired, age-related pathologies.

\section{"Poverty, infectious diseases, social imbalance, political instability, warfare, man-made and natural disasters, sanitation, and hunger and malnutrition conspire to limit the translation of the new biology into algorithms for disease prevention in the most vulnerable global populations."}

Applied in the management of degenerative diseases, the rapidly developing regenerative medicine armamentarium promises significant human health benefit with tangible outcomes for increased quality of life and improved patient care, building on breakthroughs in stem cell biology paired with successes in transplant medicine. Insights into the regenerative basis of cell, tissue and organ function, and their interface with the environment will increasingly define disease risk, identify processes mediating disease susceptibility or target mechanism-based therapies, thereby providing previously unanticipated opportunities for patient-specific disease management. Therefore, the emerging field of regenerative medicine will grow in conjuncture with the realization of the individualized medicine paradigm to create predictive, personalized and preemptive solutions for tailored patient-specific strategies. However, maximizing potential return mandates an integrated roadmap across the translational continuum to ensure optimal application of regenerative medicine algorithms in practice.

\section{Translational continuum}

Advancing the benefits of fundamental discoveries in the laboratory to patients and populations encompasses multiple integrated steps [15]. From the initial T0 phase, typically defined as discovery research, through $\mathrm{T} 1$ research, which develops a candidate health application (e.g., test or therapy), to T2 research, which evaluates a candidate application and develops evidence-based recommendations for everyday clinical practice and health decision-making, and, finally, T3 translation, which assesses how to integrate and disseminate an evidence-based recommendation into care integral to community practices. Beyond incorporating evidence-based knowledge into clinical practice, T4 translation seeks to advance scientific knowledge past algorithms for treating established disease to paradigms of prevention and behavioral alterations in communities and populations. It is at this stage of translation that the enterprise undergoes an evolution from the medical model of 'intervention' to the public health model of disease management or 'prevention'. The public health model focuses on information and education programs that eliminate deleterious behaviors at the community and population levels, and produce disease susceptibility [16]. In essence, T4 research seeks to move health practices established by the T0-3 process to directly impact the health of populations, associated with improved disease prevention and reduced costs for medical care [17]. Ultimately, the impact of these paradigms must extend beyond individual communities, to translate the maximum benefits of scientific and medical innovation to populations at large. In this context, a proposed T5 translation step goes beyond the public health model of care, extending to the social health model, which focuses on improving the health of populations by reforming suboptimal social structures $[8,16]$. Indeed, fundamental societal issues that eclipse limitations in healthcare delivery restrict the distribution of the products of scientific innovation to those populations 
in greatest need. Poverty, infectious diseases, social imbalance, political instability, warfare, man-made and natural disasters, sanitation, and hunger and malnutrition conspire to limit the translation of the new biology into algorithms for disease prevention in the most vulnerable global populations.

\begin{abstract}
"Molecular sciences, including the evolving science of biomarkers, have catalyzed the development of diagnostic and therapeutic platforms tailored to the individual patient profile, paving the way for the deployment of individualized medicine approaches that address human variation and individualized medical need."
\end{abstract}

\section{Translational teams}

The breadth and depth along the continuum of clinical and translational science mandates that no specialist has the complete knowledge base to bring new discoveries from the laboratory to populations. This translation is best accomplished by teams of investigators across different communities, with knowledge domains that are complementary and synergistic [17]. These teams will, by necessity, be comprised of members who are scientists, clinician investigators and practitioners. Furthermore, they will benefit from experts in epidemiology, informatics, regulatory knowledge, ethics, community engagement and health services research. They will be facilitated by electronic structures that efficiently link patients, providers, databases and operational resources into integrated clinical and research data allowing for efficient and streamlined deployment to address high-priority translational questions with the potential to advance the health of populations. Furthermore, the full spectrum of translation, from discovery to disease prevention, in populations, will be best served by integrating representatives from the community into investigational teams.
Community integration establishes a basis for bidirectionality in translational research, in which neighborhood representatives shape the research agenda and inform the prioritization of questions, individualizing medical research that is aligned with specific community needs. Ultimately, translational teams must incorporate investigators with knowledge domains that extend beyond the laboratory and clinic, engaging political and social scientists, engineers, economists, anthropologists and population biologists to allow the definition of the critical path that maximizes investments in research for the good of the global village. The full potential of the revolution in clinical and translational science will only be realized in the context of an associated revolution in social and political science that creates secure environments for all individuals, provides global access to safe and meaningful employment, economically enables the pursuit of healthy behaviors, and provides equal access to education and healthcare [16]. Therefore, the revolution in translational medicine has accelerated the development and application of scientific discovery and technology into patient-centered algorithms that improve the health of communities and populations. Sustained engagement of translational teams that bridge communities of patients and practice will drive and amplify innovation in the evolution of healthcare solutions.

\section{Financial \& competing interests disclosure}

A Terzic is the Marriott Family Professor of Cardiovascular Research at the Mayo Clinic. SA Waldman is the Samuel MV Hamilton Endowed Professor of Thomas Jefferson University. The authors are supported by grants from the NIH. The authors have no other relevant affiliations or financial involvement with any organization or entity with a financial interest in or financial conflict with the subject matter or materials discussed in the manuscript apart from those disclosed.

No writing assistance was utilized in the production of this manuscript.

\section{Bibliography}

1 Feero WG, Guttmacher AE, Collins FS: Genomic medicine - an updated primer. N. Engl. J. Med. 362, 2001-2011 (2010).

2 Waldman SA, Terzic A: Molecular therapeutics from knowledge to delivery. Clin. Pharmacol. Ther. 87, 619-623 (2010).

3 Hamburg MA, Collins FS: The path to personalized medicine. N. Engl. J. Med. 363, 301-304 (2010).
4 Waldman SA, Kraft WK, Nelson TJ, Terzic A: Clinical pharmacology: a paradigm for individualized medicine. Biomark. Med. 3, 679-684 (2009).

5 Arrell DK, Terzic A: Network systems biology for drug discovery. Clin. Pharmacol. Ther. 88, 120-125 (2010).

6 Terzic A, Perez-Terzic C: Channelopathies: decoding disease pathogenesis. Sci. Transl. Med. 2, 42ps37 (2010).
7 Waldman SA, Terzic A: Translational medicine in the era of health care reform. Clin. Transl. Sci. 2, 96-97 (2009).

8 Waldman SA, Terzic A: Clinical and translational science: from bench-bedside to global village. Clin. Transl. Sci. 3(5), 254-257 (2010).

9 Terzic A, Nelson TJ: Regenerative medicine advancing health care 2020. J. Am. Coll. Cardiol. 55(20), 2254-2257 (2010). 
10 Nelson TJ, Behfar A, Terzic A: Strategies for therapeutic repair: the ' $\mathrm{R} 3$ ' regenerative medicine paradigm. Clin. Transl. Sci. 1, 168-171 (2008).

11 Nelson TJ, Behfar A, Terzic A: Stem cells: biologics for regeneration. Clin. Pharmacol. Ther. 84(5), 620-623 (2008).

12 Nelson TJ, Behfar A, Yamada S, Martinez-Fernandez A, Terzic A: Stem cell platforms for regenerative medicine. Clin. Transl. Sci. 2(3), 222-227 (2009).

13 Cortese DA: A vision of individualized medicine in the context of global health. Clin. Pharmacol. Ther. 82, 491-493 (2007).
14 Waldman SA, Terzic A:

Pharmacoeconomics in the era of individualized medicine. Clin. Pharmacol. Ther. 84, 179-182 (2008).

15 Khoury MJ, Gwinn M, Yoon PW, Dowling N, Moore CA, Bradley L: The continuum of translation research in genomic medicine: how can we accelerate the appropriate integration of human genome discoveries into health care and disease prevention? Genet. Med. 9, 665-674 (2007).

16 Brook RH: Medical leadership in an increasingly complex world. JAMA 304, 465-466 (2010).
17 Kon AA: The Clinical and Translational Science Award (CTSA) Consortium and the translational research model. Am. J. Bioeth. $8,58-60$ (2008).

\section{Websites}

101 US Department of Health and Human Services. 2020: A New Vision - A Future for Regenerative Medicine www.hhs.gov/reference/newfuture.shtml

$102 \mathrm{NIH}$ http://stemcells.nih.gov/info/

103 National Academies http://dels.nas.edu/bls/stemcells/basics.shtml 